\title{
Insights for touchscreen interface guidelines for medical devices: A case study of a usability test
}

\author{
Kamya Nagarajan1, Arlindo Silva ${ }^{1}$ \& Hyowon Lee ${ }^{1}$ \\ ${ }^{1}$ Singapore University of Technology and Design \\ kamya_nagarajan@mymail.sutd.edu.sg
}

\section{Introduction}

\subsection{Usability of medical devices}

Usability is defined as "the extent to which a product can be used by specified users to achieve specified goals with effectiveness, efficiency and satisfaction in a specified context of use" (ISO 9241-11). Users in general are becoming less tolerant to poor design. In the context of medical device use, with a faster pace of patient treatment, the opportunity for use error is increased, placing further emphasis on the need for manufacturers to design devices to take such pressures into account(Ward and Clarkson 2004).

Many adverse events in medicine are the result of poor interface design rather than human error(Fairbanks and Caplan 2004). In a medical setting, user groups are diverse, and so are the use cases. Clinicians, although considered to be expert users of medical devices, have a special need to interact concurrently with the on-screen UI(User Interface) and those physical parts the device may have, while paying attention to the patients. Use error caused by inadequate medical device usability has become an increasing cause of concern (ISO/IEC 62366-1:2015). Due to rising instances of user interface-related accidents and other incidents, FDA (U.S. Food and Drug Association) has begun to include human factors and usability engineering reviews in their approval process. In medical device design, the usability engineering process is intended to identify and minimize use errors and thereby reduce use-associated risks (ISO/IEC 62366-1:2015).

\subsection{Touchscreen in medical devices}

Software controls are steadily replacing hardware controls across products, including in medical product domains. The reasons include flexibility, ability to handle variations in skills, language, functions, componentry and condition of use (Wilcox, 2005). Users possess mental models which include the models from products they use frequently. The prevailing touchscreen interfaces that users often encounter are personal hand-held devices and it appears to be a natural choice to inherit knowledge from hand-held devices while designing a touchscreen interaction in medical devices. The concepts and methods from the Human-Computer Interaction (HCI) and usability have a key role in healthcare. However, the requirement of any design is to fit the particular purpose. Designing such devices is often dependent on the skills and expertise of the designer and his or her view of the device users.

With an increased number of devices in the market that are used by patients only in nonclinical environment, the device GUI (Graphic User Interface) must be straight forward and simple to use, without requiring extensive training (Altia Inc.).

Despite the popular HCI guidelines and wide ranging literature, there are numerous studies on various UI that are found to be confusing and error prone. In a medical setting, these could lead to life-threatening situations. Medical device software development are regulated under ISO-62304, and hence it is critical that device software meets those criteria. 
Unlike mobile apps which uses graphic oriented language such as Java, C++ and HTML5, medical devices are typically programmed in C. It is also to be considered that the device must be absolutely perfect and bug free before it can be submitted for its one- or two-year long FDA approval (Altia Inc.)

\subsection{Usability test}

According to International Electrotechnical Commission, IEC 62366-1:2015, a usability test is a method for exploring or evaluating a user interface with intended users within a specified intended use environment. Usability testing is an important Human Factors Engineering methodology for healthcare industry. The main objective of a usability test is to identify as many usability problems as possible to enable design improvement. Observations made during the usability test are recorded in detail in the usability test report. These observations are usually about test details such as user groups, tasks, their outcomes. The usability test report is a salient source of information for developing usability design guidelines as the usability problems identified come directly from the target user groups.

\section{Background}

Morita et al., (2016), compared the usability of 4 critical care ventilators from market leaders with 48 critical care respiratory therapists with 16 tasks on each ventilator. This study evaluates quantitatively the usability of the ventilators with touchscreens using NASA-TLX and PSSUQ scores. They mention that the qualitative data collected in the study indicates that the choices of interaction model of each ventilator (e.g., how to select information on the screen, adjusting settings and confirming) seem to interfere with the task completion and affect the users' overall perception of the devices. But, they do not detail on those qualitative information to learn from the touchscreen interface problems on the existing devices.

Zhang et al. (2019) analyzed data from the U.S. Food and Drug Administration (FDA) database related to the recall cases of UI software errors and came up with 20 categories of UI software errors. They report that as the data was specifically from FDA database alone, it may mis-represent the overall and typical usability problems on medical devices in general and that there is a need to advance their work to help medical device manufacturers develop more effective UI.

Furniss et al., 2014, highlight through their study with an infusion pump pre-alarm problem that there is a need for HCI to develop concepts, models and tools that can more readily capture how the interactions at different levels of socio-technical system impact on the medical device

design and use. As Ward and Clarkson (2004) note in an analysis of medical device-related errors, in the context of devices such as defibrillators and blood glucose meters, devices are being used in an increasingly wide range of settings, and thus we cannot always assume that a device user will have a certain level of training, skill or physical, social or cognitive ability.

Acharya et al. (2010), in their case study on hospital bed user interfaces, found that the design of hospital bed control panels violate basic and well-known HCI principles. They also mention the below points, and to date, these points do not seem to contradict much with the current situation considering the amount of literature available in this topic.

- HCI practitioners appear to be avoiding an important area; equally, the people working in the area seem unaware of HCI. 
- HCI rarely discusses error and negative outcomes; much of the literature is about positive developments.

- In the healthcare domain, most of the literature concentrates on clinical sequelae of adverse events not on the design or $\mathrm{HCI}$ issues that created the latent conditions for the error.

- Adverse events are rarely explored from any perspective other than their clinical implications.

Bitkina et al., (2020), in their review study on medical device usability mentions one of the future ergonomic challenges in medical technology as user-product interaction and more research is needed on the interaction between user and the medical device, as in the HCI field.

Having a set of touchscreen interface guidelines considering knowledge in $\mathrm{HCI}$ and other domain, tailoring it specific to medical device applications, not only helps designers to consider all the relevant factors that influence the design, but also helps as a tool for medical device evaluation in hospitals in procurement decision-making (Ginsburg 2005; Fairbanks and Caplan 2004). The rationale behind this paper comes from the framework proposed in Tailor-made Human Factors and Usability guidelines by Nagarajan \& Silva (2019), where usability testing reports are one of the internal sources of information that could contribute to develop Human Factors and Usability guidelines for medical device design(Fairbanks and Caplan 2004).

Some device development processes may accommodate multiple instances of usability testing, while some may not; possible reasons for this lack of testing could be timeline or budget. The disadvantage of not being able to conduct multiple instances of usability testing, however, must not compromise device usability and learning from usability problems. This paper is an attempt to bridge the gap between designers and users, by including the context of various tasks in the usability testing of a medical device touchscreen.

\section{Method}

Observation of outcomes from a usability test conducted for a medical device product with touchscreen interaction was used as the source of analysis reported in this study. The usability testing was conducted with 72 participants in diverse target user groups, namely clinicians(age: 22-65), caregivers(age: 22-75) and patients-as-users(age:15-75). The testing environments considered were a clinical and a home-use set-ups. The users were assigned specific tasks to perform a therapy using the medical device in different modes. The evidences from the outcomes that mainly relate to the use of a touchscreen UI of the device were considered for this study. The qualitative information in the notes/reports was analysed using thematic analysis and the insights drawn from multiple interface-related observations. The tasks were simulated in the device to verify the observation. Among several tasks assigned to the users, those tasks which contributed to usability-related improvement opportunities alone are further discussed in this paper. The insights made from the analysis were confirmed with the team for any conflict or mis-understanding of the problem. Table 1 shows the tasks distribution among three different user groups. The details of the task assigned to the user group, context and the outcome are explained in the next section. 
Table 1: Task distribution among user groups

\begin{tabular}{|l|c|c|}
\hline S.No & User Group & No. of tasks \\
\hline $\mathbf{1}$ & Clinicians & 6 \\
\hline $\mathbf{2}$ & Clinicians + Caregivers & 3 \\
\hline $\mathbf{3}$ & Clinicians + Caregivers + Patients & 10 \\
\hline
\end{tabular}

\section{Findings}

The observation and outcome of the usability testing with various touchscreen related tasks and the follow up interview information have been analysed to derived as insights. These insights are shaped in the form of learnings and in this section we summarise each insight with the context, user group, use case and their outcome. The insights derived are grouped into 3 categories of touchscreen user interface. The 3 categories are: A) Visual Cues/Affordances; B) Error prevention; and C) Consistency. Each individual learning point is given a number and parked under one of the categories.

\section{A. Visual Cues/Affordances}

This category relates to many available general design principles such as Norman's affordance (Norman, 2013) and other usability guidelines such as Shneiderman's 8 Golden Rules (Shneiderman, et al., 2016). However, the use context of medical touchscreen UIs means some aspects of these knowledge base are more significant than others (see Section 5 on this).

Device users may be familiar with the operating sequences or control panel arrangement of the previous device design, but may assume immediately that the new device operates in the same manner(Ward and Clarkson, 2004). Difficulty in interpreting the values, unit and information is at times confusing even for trained users(Hubble et al,2000). Fairbanks and Caplan, 2004, mention this category during interface problem in their fictional scenario, but a compilation of true events reported by Emergency Medicine Providers. The inferences below warrants far more detailed considerations under this category.

1. Consider user's mental model with previously used similar medical devices and provide cues in touchscreen to remind unfamiliar actions to be performed to complete a task.

Use case: A setting to be turned $\mathrm{ON}$ on the touchscreen interface and a physical connection to be done before starting a therapy.

User group: Clinicians, caregivers and patients.

Context: The users were used with similar device which comes with integrated physical connection.

Outcome: Users forgot to connect the physical connection after turning ON the setting in touchscreen and the therapy started with incomplete connection.

2. Avoid the use of abbreviation and numerical symbols together in same information text format.

Use case: Interpret the parameter related information on screen.

User group: Clinicians.

Context: Users are to be informed about maximum value of parameter while setting the parameter.

Outcome: Users had difficulty to interpret the given information. 
3. Provide cues to align settings on touchscreen and physical parts that interact with the device.

Use case: Users are to administer a therapy with a nebulizer.

User group: Clinicians, caregivers and patients.

Context: The users have to turn nebulizer (a piece of medical equipment that can be used to administer medication directly and quickly to the lungs) ON in touchscreen interface and make a couple of physical connections to successfully complete the task.

Outcome: Users forgot to connect one of the physical connections (with device) and started the therapy.

4. Place buttons in-line with action/task sequence to complete all the steps required to complete the task.

Use case: Users are to set multiple parameters with different interface types.

User group: Clinicians.

Context: Users need to remember to set numerical values with different units, categorical input, mode changes.

Outcome: Users entered wrong values, missed changing some options required before starting the specified therapy.

\section{Consider a clear indication for user-maintained mode.}

Use case: Users need to tap and hold two on-screen buttons at different times while administering a therapy.

User group: Clinicians and caregivers.

Context: Users face divided attention between patient, i.e., holding mask and controlling a parameter in touchscreen interface.

Outcome: Users had no idea how to control the specified parameter using user-maintained mode (i.e. tap and hold instead of tap once in order to get the desired outcome) and were trying multiple options to figure out.

6. Provide clear labels of parameter along with units in input fields.

Use case: Users are to set prescribed parameters on touchscreen.

User group: Clinicians and caregivers.

Context: The values are prescribed in terms of parameter names, but the touchscreen interface has fields along with units in close proximity.

Outcome: Users had confusion relating units with parameter names.

7. Clearly differentiate START, PAUSE and STOP options as state-transition of button functions can go un-noticed while working on physical task or focussing on patients concurrently.

Use case: While the therapy is going on, users are to PAUSE the therapy to attend any interruption.

User group: Clinicians, caregivers and patients.

Context: START button transitions to PAUSE button after therapy starts(button colour changes), while users focus on patient.

Outcome: Users pressed STOP button instead of PAUSE button and the therapy stopped unintentionally.

8. Place related parameters' input fields in proximity.

Use case: Users to turn $\mathrm{ON}$ a couple of options and set parameter values accordingly. 
User group: Clinicians, caregivers and patients.

Context: One of the options and its corresponding parameters were placed together in top, and another placed apart below.

Outcome: Users missed setting up second option, and hence its following sub-parameters.

\section{Provide cues in touchscreen interface for connecting the device with any external device} to be used in parallel.

Use case: Users are to monitor patient parameter with external device.

User group: Clinicians, caregivers and patients.

Context: An external device is to be connected with the device to enable monitoring.

Outcome: Users forgot to connect external device and started therapy, hence they did not monitor patient parameter.

\section{B. Error Prevention}

"Preventing errors" is one of the usability criteria (as in Nielsen, 1993) commonly used to prioritise before starting an interaction design project, and regularly appears in general design guidelines. Tortsten Gruchmann states design based sources of error are numerous and not only can lead to lessened safety of use, but also to decreased user-friendliness. Possible sources of error in medical devices are also stated (Miniati, Iandaza, and Dori 2004). To avoid the userdriven errors in the use of medical devices, the inferences below warrants far more detailed considerations.

10. If there are similar parameters in different therapy modes or care settings, make them clear to differentiate, and their corresponding changes are unrelated.

Use case: Users are to set therapy parameters as prescribed and start the therapy.

User group: Clinicians and caregivers.

Context: The screen labels can be confusing as users come across almost similarly-labelled parameters (for example, two different types of pressure), and the task involved parallel physical attention.

Outcome: Users did not enter correct values, in some cases interchanged the values of two different parameters.

11. Provide cues to discover input errors before starting a therapy-providing therapy summary could be useful.

Use case: Users are to input prescribed therapy parameter values correctly before starting a therapy.

User group: Clinicians, caregivers and patients.

Context: Users set numerical values using UP-DOWN arrow buttons, and an additional keypad for input mode appears if preferred.

Outcome: Users did not realize wrong input values and started therapy with wrong parameter settings.

12. Possibility of inadvertent action while shifting from digital interaction to physical interaction - show changes in touchscreen action to ensure it was done purposefully.

Use case: Users are to set up therapy parameters including setting nebulizer to ON and start therapy.

User group: Clinicians, caregivers and patients.

Context: After setting up parameters and going through multiple steps in the touchscreen, users have to shift focus on patient and physical connection which involves both hands. 
Outcome: Inadvertent action occurred. Nebulizer mode was turned OFF in touchscreen while performing another action and was not realized by users.

13. Provide cues to avoid the mistake of interchanging two similar kind of input values using same input mode.

Use case: Clinicians are to scan barcodes of self and patient in separate fields.

User group: Clinicians.

Context: Although the labels are distinct for patient and clinician, input types are similar.

Outcome: Users scanned barcode of patient and clinician barcode interchanged, and other users scanned clinician barcode for both input fields.

\section{Enable automatic device lock when necessary.}

Use case: Users are to set pre-set parameters and lock the device.

User Group: Clinicians.

Context: Pre-set therapy mode is used to set parameters by clinician as prescribed for the patient and the device is sent for home care.

Outcome: Clinicians forgot to lock the clinical access after setting pre-set parameters.

\section{Allow users to set all parameters required for therapy, before starting the session.}

Use case: Users are to change flow setting.

User group: Clinicians, caregivers and patients.

Context: Flow setting was done after all the other parameter was set and the option was available while running the therapy.

Outcome: Users were unclear on how to change flow setting and he/she could not find it in the same screen as other parameters, while a START therapy button was available on the parameters screen.

16. Visibly differentiate therapy time with other time (or clock) on the screen.

Use case: Users are to start a type of therapy by pressing START button and a particular therapy option.

User Group: Clinicians, caregivers and patients.

Context: The total timer was placed in a prominent place and close to an option for starting a particular therapy.

Outcome: Users saw the main timer running and presumed as the therapy timer assuming the therapy is in process.

17. Confirm before proceeding with default values, if available.

Use case: Set required therapy parameters for homecare use.

User Group: Clinicians.

Context: This task involved multiple interaction for input values and selections.

Outcome: Users inadvertently left some parameters to default values.

\section{Consistency}

Appearing in virtually all available general design principles and guidelines (e.g. Shneiderman's many design guidelines for organising visual display, data entry (Shneiderman, et al., 2016), Nielsen's Heuristics (Nielsen, 1993), and other HCI textbooks (Sharp, et al. 2019), consistency is considered the most important principle to adhere to. The importance of internal and external consistency in user interface across applications in relationship with user's mental model has been studied by John W. S Atzinger and Lorne Olfman, 1998 and states that 
"Learning an inconsistent application will not only be more difficult, but might also result in inaccurate mental models of other applications". Improvement opportunity related to consistency and the inferences below warrants far more detailed considerations.

\section{Use same terminologies in touchscreen interface and in naming physical parts of the device.}

Use case: Users are to realise the missed physical connection and take action accordingly.

User group: Clinicians, caregivers and patients.

Context: There was a caution message to remind a physical connection and the terms used in caution message was different from the label on physical part.

Outcome: Users interpreted the caution message wrongly and had no idea what to do.

\section{Follow standard representation of time to avoid confusion with therapy prescription of} physician.

Use case: Users are to set 2.5 minute for a type of therapy time.

User group: Clinicians.

Context: Clinician receive prescription in decimals (2.5 minutes mean 2 and a $1 / 2$ minute).

The time format in interface was in mm.ss instead of mm:ss.

Outcome: Users were confused and set $2 \mathrm{~min} 50 \mathrm{sec}$ instead of $2 \mathrm{~min} 30 \mathrm{sec}$.

\section{Discussion}

A large part of the insights summarised above have much in common with those general design guidelines and heuristics available today in HCI textbooks. However, they are meaningful in the sense that they were drawn specifically from the usage of a UI as part of physical medical devices in the context of a user dealing with a patient while using the device. To prevent use error during device operation, certain factors which are occurring in the medical device context of use are to be considered in early design stages of touchscreen interfaces. Some of the insights mentioned in the paper can be compressed under the umbrella of guidelines for software-user interface mentioned in ANSI/AAMI HE75:2009. But to bridge the gap between the designer and the user, there is a need of much detailed information on task, user group and context. We see our contribution as an extension of what is already available as guidelines to target those finer details of UI design.

While we have taken care to make our study accurate within the context of medical devices use, we are unable to provide more specific details on the usability test and the medical device tested in order to protect the privacy of the manufacturer and the confidentiality of the results. The wider interface design learning points are what we are concerned with, rather than specifics of the medical device which we have considered to illustrate the wider interface improvement opportunity.

The insights derived from the study are summarized in Table 2 as number of insights distributed across the three categories identified. 
Table 2. Category of insights.

\begin{tabular}{|c|c|c|}
\hline S.No. & CATEGORY & QUANTITY \\
\hline \multirow[t]{10}{*}{$\mathbf{A}$} & Visual cues/Affordances & 9 \\
\hline & $\begin{array}{l}\text { 1. Consider user's mental model with previously used similar } \\
\text { medical devices and provide cues in touchscreen to remind } \\
\text { unfamiliar actions to be performed to complete a task. }\end{array}$ & \\
\hline & $\begin{array}{l}\text { 2. Avoid the use of abbreviation and numerical symbols together in } \\
\text { same information text format. }\end{array}$ & \\
\hline & $\begin{array}{l}\text { 3. Provide cues to align settings on touchscreen and physical parts } \\
\text { that interact with the device. }\end{array}$ & \\
\hline & $\begin{array}{l}\text { 4. Place buttons in-line with action/task sequence to complete all } \\
\text { the steps required to complete the task. }\end{array}$ & \\
\hline & 5. Consider a clear indication for user-maintained mode. & \\
\hline & 6. Provide clear labels of parameter along with units in input fields. & \\
\hline & $\begin{array}{l}\text { 7. Clearly differentiate START, PAUSE and STOP options as state- } \\
\text { transition of button functions can go un-noticed while working } \\
\text { on physical task or focussing on patients concurrently. }\end{array}$ & \\
\hline & 8. Place related parameters' input fields in proximity. & \\
\hline & $\begin{array}{l}\text { 9. Provide cues in touchscreen interface for connecting the device } \\
\text { with any external device to be used in parallel. }\end{array}$ & \\
\hline \multirow[t]{9}{*}{ B } & Error-prevention & 8 \\
\hline & $\begin{array}{l}\text { 10. If there are similar parameters in different therapy modes or care } \\
\text { settings, make them clear to differentiate, and their corresponding } \\
\text { changes are unrelated. }\end{array}$ & \\
\hline & $\begin{array}{l}\text { 11. Provide cues to discover input errors before starting a therapy - } \\
\text { providing therapy summary could be useful. }\end{array}$ & \\
\hline & $\begin{array}{l}\text { 12. Possibility of inadvertent action while shifting from digital } \\
\text { interaction to physical interaction - show changes in input action } \\
\text { to ensure it was done purposefully. }\end{array}$ & \\
\hline & $\begin{array}{l}\text { 13. Provide cues to avoid the mistake of interchanging two similar } \\
\text { kind of input values using same input mode. }\end{array}$ & \\
\hline & 14. Enable automatic device lock when necessary. & \\
\hline & $\begin{array}{l}\text { 15. Allow users to set all parameters required for therapy, before } \\
\text { starting the session. }\end{array}$ & \\
\hline & $\begin{array}{l}\text { 16. Visibly differentiate therapy time with other time (or clock) on } \\
\text { the screen. }\end{array}$ & \\
\hline & 17. Confirm before proceeding with default values, if available & \\
\hline \multirow[t]{3}{*}{$\mathbf{C}$} & Consistency & 2 \\
\hline & $\begin{array}{l}\text { 18. Use same terminologies in touchscreen interface and in naming } \\
\text { physical parts of the device. }\end{array}$ & \\
\hline & $\begin{array}{l}\text { 19. Follow global representation of time to avoid confusion with } \\
\text { therapy prescription of physician. }\end{array}$ & \\
\hline
\end{tabular}

Some of the insights in Table 2 were noticeably more specific to the context of medical devices but not on other hand-held devices (see the selected ones in Table 3). These selected insights, we believe, deserve more attention since they are not generally considered in the general guidelines when specifically tackling the characteristics of medical devices use context. 


\begin{tabular}{|c|l|}
\hline Insight No. & \multicolumn{1}{c|}{ Insights from usability test outcome } \\
\hline $\mathbf{3}$ & $\begin{array}{l}\text { Provide cues to align settings on touchscreen and physical parts that interact with the } \\
\text { medical device. }\end{array}$ \\
\hline $\mathbf{7}$ & $\begin{array}{l}\text { Clearly differentiate START, PAUSE and STOP options as state-transition of button } \\
\text { functions can go un-noticed while working on physical task or focusing on patients } \\
\text { concurrently. }\end{array}$ \\
\hline $\mathbf{9}$ & $\begin{array}{l}\text { Provide cues in touchscreen interface for connecting the device with any external device } \\
\text { to be used in parallel. }\end{array}$ \\
\hline $\mathbf{1 2}$ & $\begin{array}{l}\text { Possibility of inadvertent action while shifting from digital interaction to physical } \\
\text { interaction - show changes in input action to ensure it was done purposefully. }\end{array}$ \\
\hline $\mathbf{1 9}$ & \begin{tabular}{l} 
Follow global representation of time to avoid confusion with therapy prescription value. \\
\hline
\end{tabular} \\
\hline
\end{tabular}

The major take-aways from this study for developers of touchscreen user interfaces for medical devices are:

- It is apparent that when a task requires divided attention (the UI, other physical parts of the device, and the patient) during the session, the interface design must be extra-intuitive and involve far less cognitive load than in regular UI design.

- After pressing the START button in the UI, users start focusing on the patient's response to therapy. It was observed that any expected touchscreen interaction from the user after pressing the START button was missed by the user.

- There is a need for better interaction strategies to be in place to support more intuitive interaction elements in medical device context to prevent error, for instance, a more transparent and informed indication of tap and hold (as opposed to a simple tap and untap).

- Stronger adherence to Gestalt principles (Palmer \& Rock, 1994) is required in grouping and separating related information on the UI specifically in therapy set-up, which involves setting up multiple parameters and various types of input interaction and physical connections.

- Irrespective of users' expertise, it is essential to make clear parameter labels with units in standard format.

\section{Conclusion}

Unlike many commonly used personal and hand-held devices/apps where some user-driven errors and mistakes can be easily tolerated (e.g. pressing a wrong button to play music, inadvertently swiping an object in a game), the repercussions of use error could be severe and even fatal in some medical device usage situations. This forces us to re-think and re-prioritise the general design principles and guidelines typically practiced for touchscreen interaction today. In the medical device domain, past studies say that some medical device interface designs violate some basic HCI principles. Lessons learned from past projects' usability test could be a feed for tailoring user interface design guideline for medical devices. There are too few studies published on medical device touchscreen user interface lessons learned. In this paper, we attempt to bridge the gap between designers and users by sharing the touchscreen GUI insights generated from a usability test of a medical device as a part of that device's development process. The testing involved three different user groups- Clinicians, caregivers and patients. The test observations were analysed for lessons-learned opportunities to improve device usability. We derived insights under 3 categories: visual cues/affordances, error prevention, and 
consistency. Among 19 insights discussed in this paper, 5 of them were considered critical, and involved concurrently performing physical sub-tasks while interacting with the touchscreen. These tasks are the ones which are to be taken into account when designing medical device touchscreen user interfaces, as these are not captured appropriately by the more general guidelines for HCI.

\section{References}

A Roadmap for Designing and Deploying Medical Device GUIs - Industry Whitepaper from Altia Inc. USA.

https://www.altia.com/downloads/whitepapers/Roadmap for Designing and Deployin g Medical Device GUIs.pdf

Acharya, Chitra, Harold Thimbleby, and Patrick Oladimeji. (2010). "Human Computer Interaction and Medical Devices." Proceedings of the 2010 British Computer Society Conference on Human-Computer Interaction, BCS-HCI 2010, 168-76. https://doi.org/10.14236/ewic/hci2010.22.

AAMI HE75:2009®2013. Human factors engineering- Design of medical devices.

Bitkina, O Vl, Hyun K. Kim, and Park, J. (2020). "Usability and User Experience of Medical Devices: An Overview of the Current State, Analysis Methodologies, and Future Challenges." International Journal of Industrial Ergonomics 76 (November 2018): 102932. https://doi.org/10.1016/j.ergon.2020.102932.

Fairbanks, Rollin J., and Stanley Caplan. (2004). "Poor Interface Design and Lack of Usability Testing Facilitate Medical Error." Joint Commission Journal on Quality and Safety 30 (10): 579-84. https://doi.org/10.1016/S1549-3741(04)30068-7.

Ginsburg, Gill. (2005). "Human Factors Engineering: A Tool for Medical Device Evaluation in Hospital Procurement Decision-Making." Journal of Biomedical Informatics 38 (3): 213-19. https://doi.org/10.1016/j.jbi.2004.11.008.

Miniati, Roberto, Ernesto Iandaza, and Fabrizio Dori. (2004). "Human Factors Engineering in Healthcare." In Clinical Engineering: From Devices to Systems, 21:167-82. Elsevier. https://doi.org/10.1201/9781315273297-15.

Ward, James R., and P. John Clarkson. (2004). An Analysis of Medical Device-Related Errors: Prevalence and Possible Solutions. Journal of Medical Engineering and Technology. Vol. 28. https://doi.org/10.1080/0309190031000123747.

IEC 62304:2006: Medical device software - Software life cycle processes," International Organization for Standardization, July 16, 2010,

ISO/IEC62366-1. (2015).“Application of usability engineering to medical devices”.

Nagarajan.K \& Silva.A. (2019). "Tailor-Made design guidelines for human factors and Usability of medical device design: A proposed methodology", Proceedings of International Conference of Engineering and Design.

Nielsen J. (1993). "Usability engineering”, Morgan Kaufman.

Norman D. (2013). "Design of everyday things" (Revised Ed.), Basic Books.

Palmer.S and Rock.I. (1994). "Rethinking perceptual organization: The role of uniform connectedness", Psychonomic Bulletin Review 1.1.29-55.

Sharp H, Preece J and Rogers Y. (2019). "Interaction design: beyond human-computer interaction" (5th Ed.), John Wiley \& Sons.

Shneiderman B, Plaisant C, Cohen M, Jacobs S, Elmqvist N and Diakopoulos N. (2018) "Designing the user interface: strategies for effective human-computer interaction" (6th Ed.), Pearson.

Ward, James R., and P. John Clarkson. (2004). An Analysis of Medical Device-Related Errors: Prevalence and Possible Solutions. Journal of Medical Engineering and Technology. Vol. 28. https://doi.org/10.1080/0309190031000123747. 
Wilcox.B.S, (2005). "Designing Usability into medical products" p11, Taylor \& Francis.

Zhang.Y, Masci.P, Jones.P, and Thimbleby.H. (2019). "User Interface Software Errors in Medical De vices: Study of U.S. Recall Data”. Biomedical Instrumentation \& Technology, 346-357. 